\title{
Molecular determinants of intrinsic resistance to doxorubicin in human cancer cell lines
}

\author{
MARZIA B. GARIBOLDI ${ }^{1}$, RAFFAELLA RAVIZZA ${ }^{1}$, LOREDANA RIGANTI ${ }^{1}$, STEFANIA MESCHINI ${ }^{2}$, \\ ANNARICA CALCABRINI ${ }^{2}$, MANUELA MARRA ${ }^{2}$, GIUSEPPE ARANCIA ${ }^{2}$, \\ ERSILIA DOLFINI $^{3}$ and ELENA MONTI ${ }^{1}$
}

\author{
${ }^{1}$ DBSF, University of Insubria, Via A. da Giussano 10, I-21052 Busto Arsizio (VA); ${ }^{2}$ Laboratory of Ultrastructures, \\ Istituto Superiore di Sanità, Viale Regina Elena 299, I-00161 Rome; ${ }^{3}$ Department of Biology and \\ Genetics for Health Sciences, University of Milan, Milan, Italy
}

Received July 17, 2002; Accepted September 11, 2002

\begin{abstract}
Intrinsic or acquired drug resistance poses a major challenge to the success of chemotherapy in the clinical management of human cancers. While acquired multidrug resistance (MDR), whereby cells become refractory to multiple drugs, has been extensively investigated, the mechanistic basis for intrinsic resistance remains elusive, so that this condition is largely unmanageable in the clinical setting. To address this issue, we have assessed the effects of the anticancer agent doxorubicin (DX) on a panel of human tumor cell lines originally derived from untreated patients and tried to establish a correlation between cell response and a number of parameters, including drug accumulation and/or drug efflux; differences in expression and/or subcellular distribution of proteins involved in the apoptotic process (e.g., p53, Bcl-2, Bax) and intracellular signal transducers (PKC $\alpha)$; changes in key detoxification processes. Based on our results, 'classic' multispecific drug transporters (P-glycoprotein, MDR-related proteins) only seem to play a minor role in the intrinsically resistant phenotype, whereas LRP may contribute to resistance in non-small cell lung carcinoma (NSCLC) cells. No relationship was observed between drug response and expression and/or subcellular localization of apoptosis-related proteins; however, increased PKC $\alpha$ levels are associated with poor drug response, suggesting that one or more substrates of this enzyme may be relevant to the resistant phenotype. Finally, overactive glutathione-recycling pathways may contribute to DX resistance.
\end{abstract}

Correspondence to: Professor Elena Monti, DBSF, University of Insubria, Via A. da Giussano 10, I-21052 Busto Arsizio (VA), Italy

E-mail: elena.monti@uninsubria.it

Key words: intrinsic drug resistance, chemotherapy, doxorubicin

\section{Introduction}

The resistance of human malignancies to multiple chemotherapeutic agents remains a major obstacle to the success of cancer therapy. Resistance is defined as intrinsic when previously untreated tumor cells fail to respond to one or more anticancer agents; when tumors initially respond to anticancer agents, but become refractory on subsequent treatment cycles, the resistant phenotype is described as acquired. It is now clear that multidrug resistance (MDR) is always multifactorial, with at least two resistance mechanisms simultaneously operating in the same tumor cell (1). These may include: i) decreased drug accumulation and/or altered intracellular distribution; ii) increased detoxification; iii) increased DNA repair; iv) altered cell cycle regulation; and v) uncoupling of the pathways linking cellular damage with programmed cell death. Resistance to a number of anticancer agents has been associated with overexpression of multispecific drug transporters, such as the $170 \mathrm{kDa}$ P-glycoprotein (P-gp) or members of the multidrug resistance-related protein family (MRPs), belonging to the ATP-binding cassette (ABC) superfamily (2-4). ABC transporters have been identified in the plasma membrane, as well as in intracellular membranes, whereby they might alter drug accumulation and/or access to nuclear targets. More recently, another protein which may be involved in drug export from the nucleus has been identified in multidrug resistant cells and indicated as LRP (lung resistance-related protein) $(5,6)$. This is a $104-110 \mathrm{kDa}$ protein not belonging to the ABC superfamily, which has been proposed to concur to nuclear pore complex formation and to transport anticancer agents away from the nucleus $(7,8)$. Alternative or additional mechanisms may contribute to the resistant phenotype, including increased activity of detoxification systems. One such system is the glutathione (GSH) system, which may or may not act in concert with drug efflux pumps; changes in intracellular GSH levels and/or in the activity of GSH-dependent enzymes (e.g., GSH transferases and GSH reductase) have been shown to be associated with resistance to several anticancer drugs (9).

A more general mechanism for drug resistance has recently emerged from preclinical observations, correlating 
the efficacy of chemotherapeutic drugs with their ability to induce apoptosis in a variety of tumor cell lines. Thus, this mode of cell death has acquired major pharmacological interest, raising the possibility that susceptibility to apoptosis induction might be a critical determinant of tumor cell response $(10,11)$. In particular, alterations of the expression or subcellular localization of proteins involved in the apoptotic machinery (such as Bcl-2, Bax, p53) have been proposed to explain the development of resistance in different cell lines. Interestingly, post-translational modifications may also play a significant role in determining the subcellular localization and/or in modulating the function of resistance-related proteins, including $\mathrm{ABC}$ transporters and proteins controlling apoptosis; the $\alpha$ isoform of PKC has been proposed as a major determinant for resistance-inducing post-translational modifications $(12,13)$.

The aim of the present study was to investigate the molecular mechanisms associated with intrinsic resistance to the widely used anthracycline agent doxorubicin (DX). A panel of five human cancer cell lines from different tissues was used, including: MCF7 breast adenocarcinoma cells, A2780 ovarian carcinoma cells, M14 melanoma cells, A549 non-small cell lung carcinoma (NSCLC) cells; and LoVo colon adenocarcinoma cells. All the cell lines were originally derived from untreated patients. We evaluated: i) the response of the cell lines to DX treatment for $120 \mathrm{~h}$; ii) the relationship between cell response, expression of drug transporters and drug accumulation; iii) the relationship between cell response and expression of proteins involved in apoptotic cell death, including p53, Bcl-2, Bax; PKC $\alpha$ levels were also assessed as it may act as a post-translational modulator for some of them. Further investigations were performed in MCF7 and A549 cells, which were the most and the least sensitive among the cell lines tested, respectively; on these cell lines we evaluated the effects of DX treatment on iv) intracellular levels and subcellular distribution of p53, Bcl-2, Bax and $\mathrm{PKC} \alpha$, and v) intracellular glutathione (GSH) levels and glutathione transferase (GST) and glutathione reductase (GSR) activities.

\section{Materials and methods}

Chemicals. 3-(4,5-dimethylthiazol-2-yl)-2,5-diphenyltetrazolium bromide (MTT) and standard reagents were purchased from Sigma-Aldrich Srl (Milan, Italy).

Cells and in vitro culture conditions. All the cell lines used in this study were obtained from ATCC (Rockville, MD), were cultured at $37^{\circ} \mathrm{C}$ under $5 \% \mathrm{CO}_{2}$ and used when in log-phase growth. The MCF7, M14, A549 and A2780 cells were maintained in RPMI 1640 medium supplemented with $10 \%$ FBS, $1 \%$ antibiotic mixture and glutamine; whereas LoVo cells were maintained in Ham's F12 medium supplemented with $10 \%$ FBS and vitamins. All cell lines used were negative to mycoplasms.

Cytotoxicity studies. The MTT assay (14) was used to assess the antiproliferative effects of DX. Briefly, cells were seeded onto 96-well plates, allowed to attach and grow for $24 \mathrm{~h}$ and subsequently exposed to DX $\left(2 \times 10^{-9}-2 \times 10^{-6} \mathrm{M}\right)$. After $120 \mathrm{~h}$,
MTT was added for $3 \mathrm{~h}$ at $37^{\circ} \mathrm{C}$. Formazan crystals, formed by mitochondrial reduction of MTT, were solubilized in DMSO and the absorbance was read at $570 \mathrm{~nm}$, using a Universal Microplate reader EL800 (Bio-Tek Instruments).

Preparation of cell extract and subcellular fractions. Total protein extracts were obtained by incubation of $10^{7}$ cells with $60 \mu \mathrm{l}$ of lysis buffer ( $1 \% \mathrm{NP} 40,10 \mu \mathrm{g} / \mathrm{ml}$ leupeptin, $10 \mu \mathrm{g} / \mathrm{ml}$ aprotinin in PBS).

To obtain subcellular fractions, $6 \times 10^{7}$ cells per sample were washed twice in ice-cold PBS, resuspended in $2 \mathrm{ml}$ of hypotonic buffer ( $5 \mathrm{mM}$ Tris $\mathrm{pH} 7.4,5 \mathrm{mM} \mathrm{KCl}, 1.5 \mathrm{mM}$ $\mathrm{MgCl}_{2}, 0.1 \mathrm{mM}$ EGTA, $1 \mathrm{mM}$ DTT) containing $0.2 \mathrm{mM}$ PMSF, $5 \mu \mathrm{g} / \mathrm{ml}$ leupeptin, $5 \mu \mathrm{g} / \mathrm{ml}$ aprotinin, $0.7 \mu \mathrm{g} / \mathrm{ml}$ pepstatin $\mathrm{A}$, and incubated for $30 \mathrm{~min}$ on ice. After homogenization, samples were centrifuged at $500 \mathrm{x} \mathrm{g}$ at $4^{\circ} \mathrm{C}$ to obtain the nuclear fraction $(\mathrm{N})$. The resulting supernatant was centrifuged at $10,000 \times \mathrm{g}$ at $4^{\circ} \mathrm{C}$ to obtain the $\mathrm{HM}$ fraction (heavy membranes, mostly consisting in mitochondria) and the supernatant from this centrifugation was then centrifuged at $150,000 \mathrm{x} g$ to obtain the LM (light membrane) and cytosolic (C) fractions. Nuclear, HM and LM fractions were resuspended in $0.1 \mathrm{ml}$ of Triton X-100 lysis buffer (1\% Triton X-100 in PBS, $5 \mu \mathrm{g} / \mathrm{ml}$ leupeptin, $5 \mu \mathrm{g} / \mathrm{ml}$ aprotinin, $0.2 \mathrm{mM}$ PMSF). Protein concentration in whole cell extracts and subcellular fractions was determined by the bicinchoninic acid (BCA) assay (Pierce, Rockford, IL, USA).

Immunoblotting. Western blot analysis was performed to detect the levels of $\mathrm{p} 53, \mathrm{Bax}, \mathrm{Bcl}-2$ and $\mathrm{PKC} \alpha$ in protein extracts from untreated cells and the expression of the same proteins in subcellular fractions from control and DX-treated samples $(2 \mu \mathrm{M}$ for MCF7 cells and $50 \mu \mathrm{M}$ for A549 cells for $24 \mathrm{~h}) .10 \mu \mathrm{g}$ of protein per lane were loaded onto polyacrylamide gels (11\%) and separated under denaturing conditions. Protein bands were then transferred onto Immobilon $\mathrm{P}$ membranes (Millipore, Bedford, MA, USA), and Western blot analysis was performed by standard techniques with mouse monoclonal antibodies (Santa Cruz Biotechnology, Inc., Santa Cruz, CA, USA) recognizing human Bcl-2 and p53 (DO-1), with a rabbit anti-PKC $\alpha$ monoclonal antibody (Sigma-Aldrich Srl, Milan) and with a rabbit polyclonal antibody raised against human Bax (Pharmingen Inc., San Diego, CA, USA). Protein bands were visualized using a peroxidase-conjugated anti-mouse/rabbit secondary antibody and the BM Chemiluminescence Western blot kit (Roche, Milan, Italy).

Determination of GSH, GST and GSR levels. MCF7 and A549 control and DX-treated cells $(2 \mu \mathrm{M}$ for MCF7 cells and $50 \mu \mathrm{M}$ for A549 cells) for $24 \mathrm{~h}$ were harvested with PBSEDTA, washed with PBS, resuspended at $10^{7}$ cells $/ \mathrm{ml}$ in phosphate buffer and sonicated. The supernatants obtained by centrifugation of cell sonicates at $30,000 \mathrm{~g}$ were used to determine intracellular GSH levels by the fluorymetric method described by Hissin and Hilf (15), using $o$-phthalaldehyde (OPT) as a fluorescent agent. GST activity was determined by monitoring the formation of GSH-adducts with 1-chloro2,4-dinitrobenzene (CDNB), according to the method described by Habig et al (16). GSR activity was measured by the method previously described by Colman (17). 


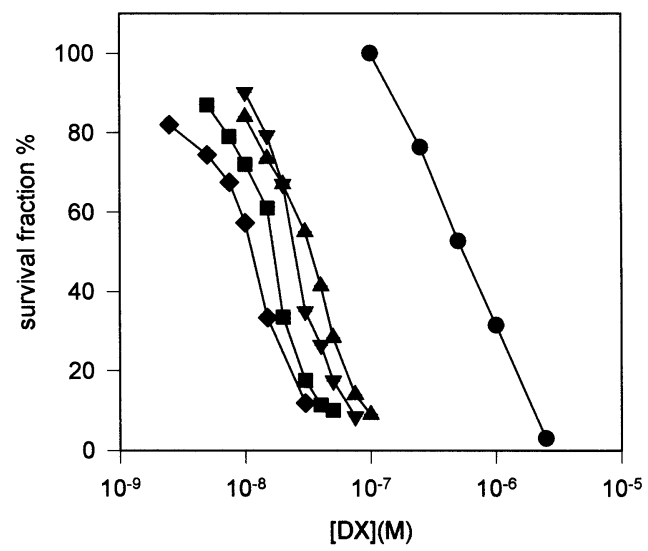

Figure 1. Dose-response curves obtained for M14 ( $\mathbf{v})$, A549 (•), A2780 (^), MCF7 ( ) and LoVo (₫) cells after 120-h continuous exposure to DX. The curves are representative of 4-6 independent experiments; values are expressed as the means $\pm \mathrm{SD}$ of 6-8 determinations/experiment.

Table I. $\mathrm{IC}_{50}$ values indicated as mean $\pm \mathrm{SE}$ of $4-6$ independent experiments.

\begin{tabular}{lcl}
\hline Cell line & $\mathrm{IC}_{50} \pm \mathrm{SE}(\mathrm{nM})$ & $\mathrm{p}<0.05$ \\
\hline M14 & $20.81 \pm 4.59$ & vs A549, A2780 \\
A549 & $514.09 \pm 51.17$ & vs MCF7, A2780, M14, LoVo \\
A2780 & $32.47 \pm 5.75$ & vs MCF7, A549, M14, LoVo \\
MCF7 & $12.78 \pm 1.79$ & vs A549, A2780 \\
LoVo & $15.20 \pm 3.16$ & vs A549, A2780 \\
\hline
\end{tabular}

Flow cytometry. Flow cytometric analysis was carried out on cell suspensions $\left(10^{6}\right.$ cells $\left./ \mathrm{ml}\right)$ obtained detaching monolayer growing cells by trypsin-EDTA solution.

To evaluate the intracellular expression of P-gp and LRP cell suspensions were fixed with $2 \%$ paraformaldehyde in PBS and the samples were then permeabilized by adding $0.05 \%$ Triton X-100. Incubations with the primary (MRK16, LRP-56, LRP-42) and secondary (goat anti-mouse IgG-fluorescein conjugate) antibodies were performed in PBS containing $1 \%$ BSA, $10 \%$ fetal calf serum and $10 \%$ human AB serum. For negative controls, cells were labeled with mouse isotypic globulins.

For MRP1 detection with MAb MRPr1, cells were permeabilized in $10 \%$ (v/v) lysing solution $\mathrm{G}$ (Becton Dickinson, Mountain View, CA) in $\mathrm{dH}_{2} \mathrm{O}$ and incubated in PBS/BSA containing $1 \%$ normal goat serum. Cells were first incubated with the MRPr1 antibody and subsequently with a goat antirat IgG fluorescein-conjugate. For negative controls, cells were incubated with rat isotypic globulins.

Time course studies of drug accumulation were performed on cells treated with $2 \mu \mathrm{M}$ DX for $1 \mathrm{~h}$ and $4 \mathrm{~h}$. At the end of treatments, cells were washed with ice-cold Hank's balanced salt solution (HBSS, Sigma), detached with EDTA and trypsin, resuspended in ice-cold PBS and immediately analyzed for
A

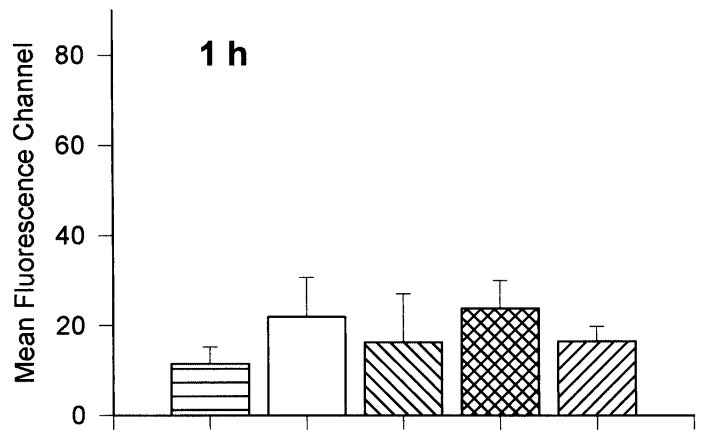

$\mathbf{B}$

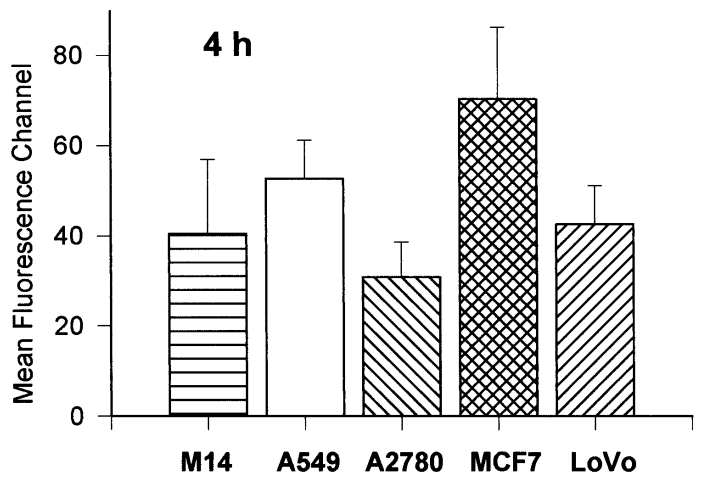

Figure 2. DX accumulation in the five cell lines considered after 1 and $4 \mathrm{~h}$ of treatment. Values are expressed as mean \pm SD of 3-5 independent experiments.

DX content. Fluorescence was analyzed with a FACScan flow cytometer (Becton Dickinson) equipped with a $15-\mathrm{mW}$, $488 \mathrm{~nm}$, air-cooled argon ion laser. Fluorescence emissions were collected through a $530 \mathrm{~nm}$ band-pass filter for fluorescein and a $575 \mathrm{~nm}$ band-pass filter for DX and acquired in $\log$ mode. For DX accumulation studies, drug fluorescence intensity was expressed as the mean fluorescence channel (MFC) calculated from the flow cytometric profiles by the Cell Quest software. For quantitative evaluation of P-gp, MRP and LRP expression, protein content in arbitrary units (A.U.) was expressed as the ratio of the MFC of labeled samples on the MFC of negative controls.

\section{Results}

Cytotoxicity studies. Fig. 1 shows representative dose-response curves obtained after 120 h exposure of A549, A2780, M14, MCF7 and LoVo cells to increasing concentrations of DX. $\mathrm{IC}_{50}$ values, determined according to the median effect equation (18) and indicated as mean \pm SE of at least 4 independent experiments, are reported in Table I. Of all the cell lines tested, MCF7 cells were the most sensitive to DX treatment, followed by the colon cell line LoVo and M14 melanoma cells, both with comparable $\mathrm{IC}_{50}$ values; A2780 ovarian carcinoma cells exhibited a statistically significant increase in $\mathrm{IC}_{50}$ as compared to the MCF7, LoVo and M14 cells, whereas the NSCLC cell line A549 was significantly less responsive than all the other cell lines tested, with approximately a 40-fold resistance to DX treatment compared to MCF7 cells. 
A

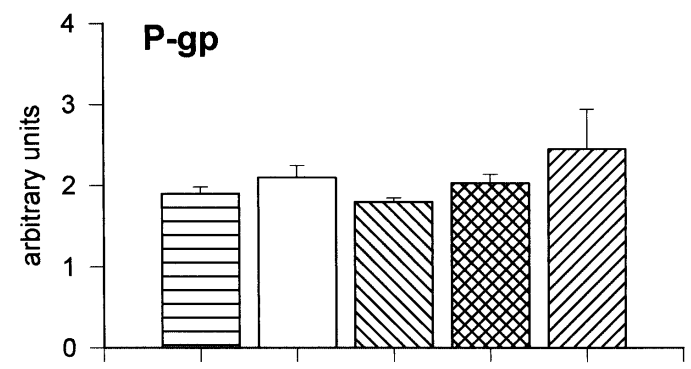

B

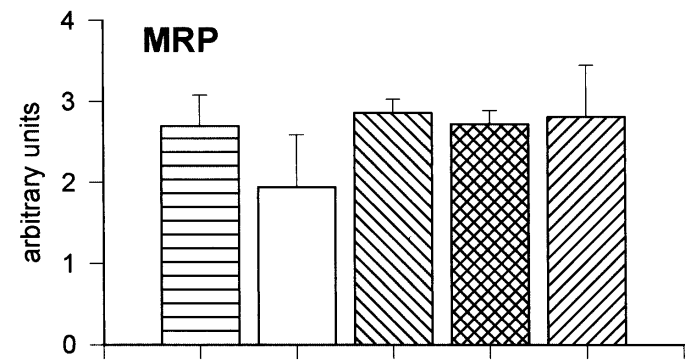

C

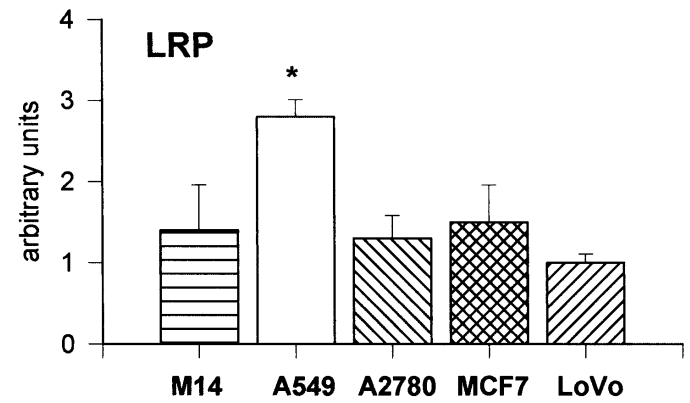

Figure 3. Flow cytometric analysis of P-gp, MRP and LRP levels in M14, A549, A2780, MCF7 and LoVo cell lines. Values are expressed as mean \pm SD of 3-5 independent experiments $\left({ }^{*} \mathrm{p}<0.05\right.$ vs the other cell lines).

$D X$ accumulation. To verify the hypothesis that a lower sensitivity to DX could be due to reduced intracellular drug concentration, drug accumulation was evaluated by flow cytometry at two different time points. Following cell exposure to $2 \mu \mathrm{M}$ DX, no significant differences in DX accumulation were observed among the cell lines considered, either after $1 \mathrm{~h}$ (Fig. 2A) or $4 \mathrm{~h}$ (Fig. 2B); therefore no significant correlation could be established between intracellular drug retention and cytotoxic effect.

P-gp, MRP and LRP expression. Fig. 3 shows the fluorescent signals measured by flow cytometry (expressed in arbitrary units), corresponding to levels of P-gp (Fig. 3A), MRP1 (Fig. 3B) and LRP (Fig. 3C) in MCF7, A549, A2780, M14 and LoVo cell lines. No significant differences in P-gp or MRP levels were observed among the cell lines considered. In contrast, the expression of LRP was found to be significantly higher in A549 cells than in the other cell lines (Fig. 3C, $\mathrm{p}<0.05$ vs the other four cell lines).

Expression of the p53, Bcl-2, Bax and PKCa. Fig. 4 shows the baseline expression levels of p53 (Fig. 4A) in total cell lysates obtained from the five cell lines considered in this study. M14 and LoVo cells exhibited the highest p53 levels,

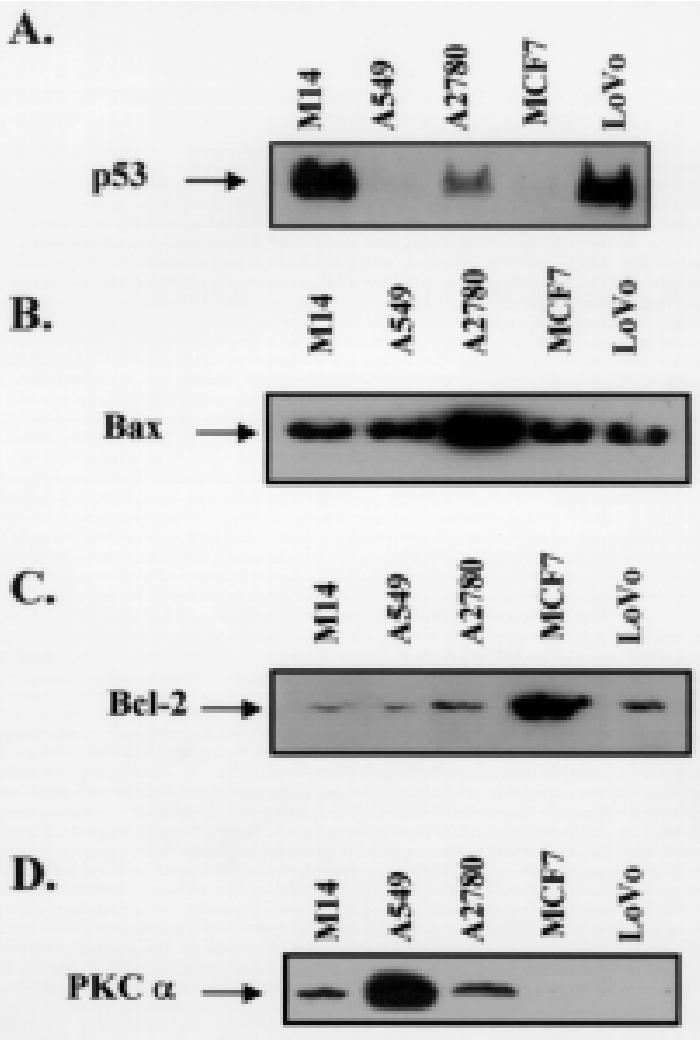

Figure 4. Western blot analysis of protein extracts from untreated cells of p53 (A), Bax (B), Bcl-2 (C) and PKC $\alpha$ (D) in M14, A549, A2780, MCF7, and LoVo cells.

while MCF7 and A549 the lowest. Bax levels are highest in A2780 cells followed by A549 and MCF7 cells and then M14 and LoVo cell lines (Fig. 4B). Bcl-2 levels are shown on Fig. 4C: MCF7 cells show the highest levels of expression, followed by LoVo, A2780, M14 and A549 cell lines. The highest PKC $\alpha$ protein levels were observed in A549, followed by A2780 and M14 cells (Fig. 4D). It is possible to conclude that no direct correlation exists between $\mathrm{p} 53, \mathrm{Bcl}-2$ or Bax protein levels and sensitivity to DX. The observation that $\mathrm{PKC} \alpha$ is undetectable in the two most sensitive cell lines (MCF7 and LoVo), present at low and intermediate levels in M14 and A2780 cells, respectively (which also exhibit low and intermediate response to DX) and significantly overexpressed in the intrinsically resistant A549 cells, suggests a possible role for $\mathrm{PKC} \alpha$ in the intrinsically resistant phenotype.

Subcellular distribution of $p 53, B c l-2, B a x$ and PKCa. Western blot analysis with anti-p53 antibody showed that in MCF7 untreated cells the protein is expressed at low levels and mainly localized in the nuclear and mitochondrial fractions. Exposure to DX induces an increase in p53 levels in all subcellular compartments (Fig. 5A). In contrast, in A549 cells exposed to DX translocation of p53 from the cytoplasm to the nucleus was observed (Fig. 5B). Fig. 6A shows an increase in Bax expression in the light membrane fraction in MCF7 cells following DX treatment, while in A549 cells Bax levels increased in all subcellular fractions except the cytoplasm after DX treatment (Fig. 6B). 


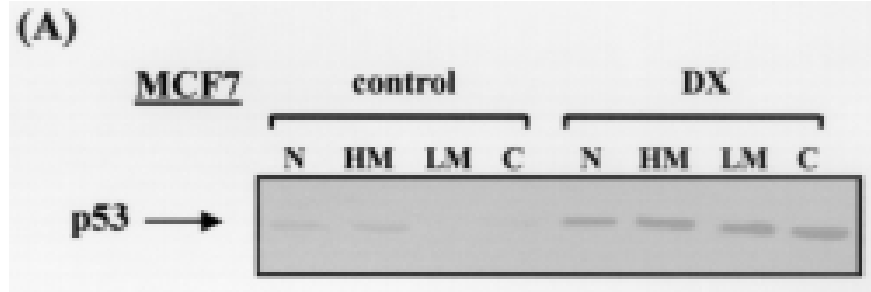

(B)

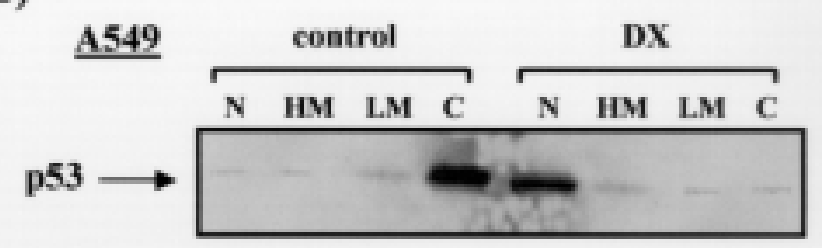

Figure 5. p53 protein levels in subcellular fractions of MCF7 and A549 cell lines with or without DX treatment $(\mathrm{N}$, nuclear fraction; LM, light membrane fraction; HM, heavy membrane fraction; $\mathrm{C}$, cytosolic fraction).

\section{A}

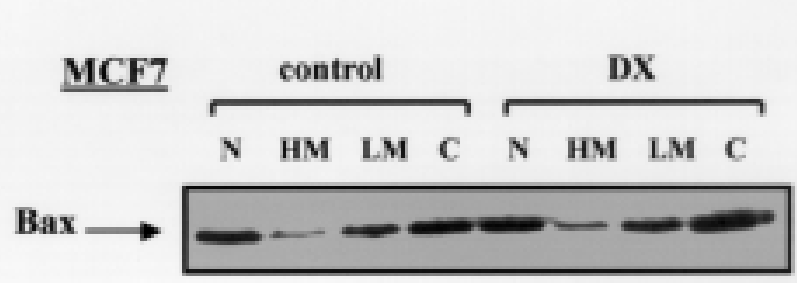

B

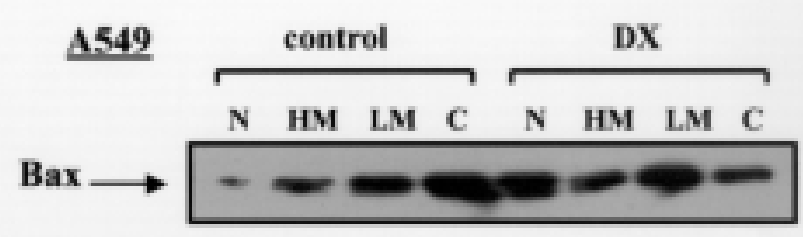

Figure 6. Bax protein levels in subcellular fractions of MCF7 and A549 cell lines with or without DX treatment (N, nuclear fraction; LM, light membrane fraction; HM, heavy membrane fraction; $\mathrm{C}$, cytosolic fraction).

Fig. 7A shows Bcl-2 subcellular distribution in MCF7 cells treated with DX; before treatment the protein is mainly present in the nuclear and mitochondrial fractions; following DX exposure, its expression increases in the cytoplasm and decreases in the light membrane fraction, whereas nuclear and mitochondrial expression is unmodified. In A549 cells, Bcl-2 levels are very low or undetectable in all the subcellular fractions examined, and DX treatment does not affect levels of expression or subcellular distribution of the protein (Fig. 7B).

$\mathrm{PKC} \alpha$ protein distribution in MCF7 cells is shown in Fig. 8A. In this cell line a generalized increase in $\mathrm{PKC} \alpha$ protein levels can be observed in all the subcellular fractions, including nuclei, following DX exposure. In contrast, A549 cells exhibit comparable levels of the protein in all the
A

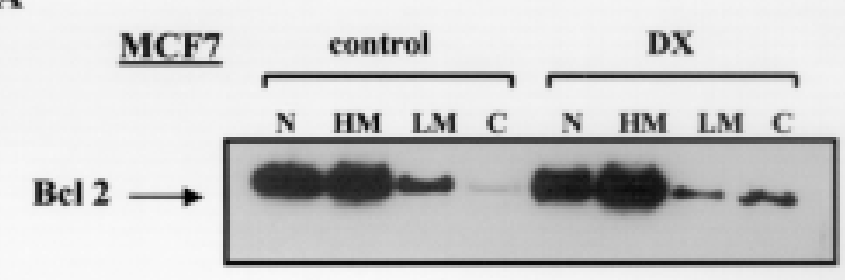

B

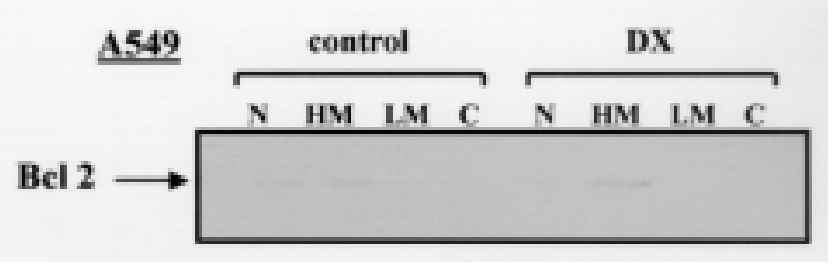

Figure 7. Bcl-2 protein levels in subcellular fractions of MCF7 and A549 cell lines with or without DX treatment $(\mathrm{N}$, nuclear fraction; LM, light membrane fraction; HM, heavy membrane fraction; $\mathrm{C}$, cytosolic fraction).

A

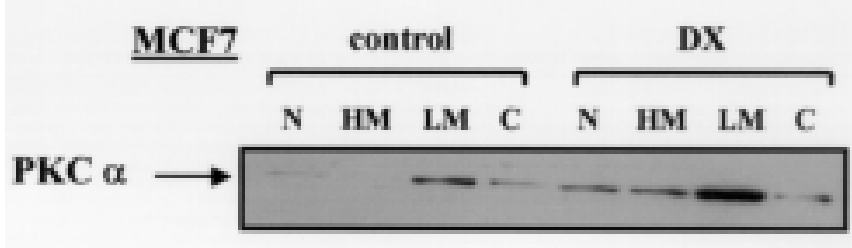

B

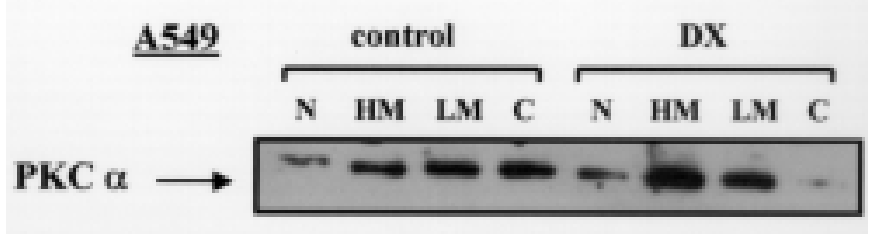

Figure 8. PKC $\alpha$ protein levels in subcellular fractions of MCF7 and A549 cell lines with or without DX treatment $(\mathrm{N}$, nuclear fraction; LM, light membrane fraction; HM, heavy membrane fraction; C, cytosolic fraction).

subcellular fractions, except the nucleus, which exhibits significantly lower levels; DX exposure induces an increase in PKC $\alpha$ levels in the light membrane fraction and a decrease in the cytoplasm, without affecting the nuclear levels of the protein (Fig. 8B).

GSH, GST and GSR levels. GSH levels were measured in MCF7 and A549 cell lines, as described in the methods section, either with or without treatment with equitoxic doses of DX. As reported in Fig. 9A, baseline GSH levels are higher in MCF7 than in A549 cells. Following DX treatment, GSH levels in MCF7 cells decreased dramatically, while the intracellular GSH pool remained unaffected in A549 cells. Baseline values of GST activity are similar in MCF7 and A549 cells 

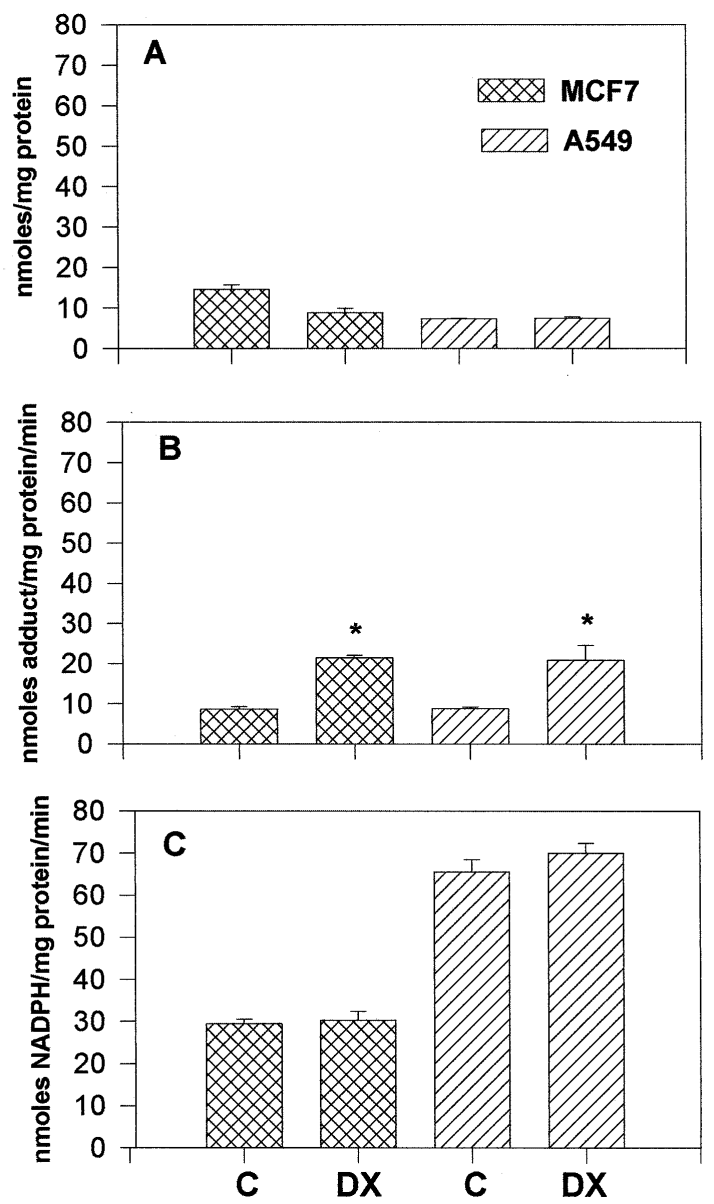

Figure 9. GSH levels (A), GST (B) and GSR (C) activities in MCF7 and A549 cells treated with equitoxic doses of DX. Values are expressed as mean \pm SD of 4-6 independent experiments ( ${ }^{*} \mathrm{p}<0.05$ vs controls).

and a similar significant increase was evidenced in both cell lines after DX treatment (Fig. 9B). In contrast, higher levels of GSR activity were measured in A549 cells than in MCF7 cells, while and a minor, non-significant increase in GSR activity was observed in both cell lines following exposure to DX (Fig. 9C).

\section{Discussion}

The ability of tumor cells to evade the effects of anticancer agents remains a major cause of treatment failure in cancer patients. The term multidrug resistance (MDR) is classically used to define a resistant phenotype whereby cells become resistant simultaneously to different drugs with no obvious structural resemblance and with different cellular target(s) (1). Different pathways have been proposed to be involved in intrinsic or acquired MDR; one of the best documented mechanisms is modulation of intracellular drug concentrations. This can be achieved through decrease in drug uptake or increase in drug efflux. At present, a number of drug efflux proteins have been characterized, including P-gp, exporting paclitaxel, vinca alkaloids, anthracyclines and other natural product-derived drugs (2,3), and the MRP family, with MRP1 and MRP2 also implicated in transport of anthracyclines and vinca alkaloids (4). In the present study, we evidenced different levels of intrinsic resistance to DX in the MCF7, A2780, LoVo, M14 and A549 cell lines, which were selected as representative of five different tumor types exhibiting varying degrees of drug responsiveness in the clinic. Differences in sensitivity did not seem to correlate with different intracellular drug accumulation, as flow cytometric analysis of this parameter did not show any significant differences among the five cell lines tested. Determination of ABC multispecific drug transporters did not show any significant differences in the expression of P-gp or MRP1, suggesting that these transporters may play minor roles in the intrinsically resistant phenotype. In contrast, significant LRP overexpression was detected in A549 cells, which were the most refractory to DX treatment. This is in agreement with recent data, showing a correlation between LRP levels and acquired resistance to chemotherapeutic treatment in cancer cell lines (5). Recent data from our group indicate that increased LRP expression in A549 as compared with MCF7 cells correlates with a different intracellular DX distribution, whereby high nuclear drug levels can be detected in breast cells, while drug-related fluorescence is confined to perinuclear vesicular structures in lung cells (19). Thus, LRP overexpression seems to reduce drug access to its molecular targets in A549 cells, even though overall drug accumulation does not significantly differ from that observed in the other cell lines tested; this may account for the relative resistance of this cell line. However, the remaining cell lines showed similar levels of this protein, even though A2780 ovarian cells were significantly less responsive to DX than MCF7, M14 or LoVo cells (Table I). Therefore, we can only conclude that, while LRP may be a critical determinant of drug resistance in lung-derived cell lines, it does not seem to play a major role in determining the intrinsically resistant phenotype in other cell lines.

Besides multidrug transporters, other pathways have been involved in resistance to chemotherapeutic, including: i) alterations of intracellular drug metabolism, either through decreased activation or increased inactivation, ii) alterations of cellular drug targets, such as point mutations in the DNA topoisomerase II gene, leading to amsacrine resistance, or in the tubulin gene, preventing paclitaxel binding, iii) enhancement of DNA repair.

Regarding detoxification mechanisms, conjugation to glutathione $(\mathrm{GSH})$ prior to export from the cell is an important detoxification mechanism for anticancer as well as other drugs; furthermore, GSH has been reported to act as a cofactor for extrusion of unconjugated agents (anthracyclines, vinca alkaloids) by MRPs $(20,21)$. Accordingly, MDR in tumor cell lines is often accompanied by an increase in cellular thiol content, in the form of GSH, and by an increase in GSHdependent enzymatic activities, such as glutathione transferase and reductase $(9,22,23)$. Comparison of GSH and GSHdependent enzymes in MCF7 and A549 cells indicates that the latter are more efficient in recycling intracellular GSH by the GSR pathway, even though GSH levels are in fact lower than in the MCF7 cells. Efficient recycling of GSH is essential in lung cells, which are naturally exposed to high levels of oxygen tension and are therefore more prone to develop oxidative stress. This property may contribute to resistance to cytotoxic insults involving generation of oxygen-derived reactive species (which is the case with DX). The increased 
levels of GST observed in A549 cells after DX treatment could also concur to the resistant phenotype, as GST levels have frequently been related to resistance to anthracycline anticancer agents $(24,25)$.

More recently, the observation that effective chemotherapeutic drugs are able to induce apoptosis has indicated susceptibility to apoptosis as a critical determinant of tumor cell response to both radio- and chemotherapy $(10,11)$.

A number of proteins able to induce or inhibit apoptosis have been identified, including p53 and members of the Bcl-2 family of proteins (11), and changes in their expression and/or subcellular distribution have been proposed to explain drug resistance in different cell lines. Our results did not show any relationship between p53, Bcl-2 and Bax protein levels and sensitivity to DX. Changes in subcellular localization of these proteins, which were observed in MCF7 and A549 cells, also fail to explain the observed difference in drug responsiveness. On the other hand, PKC $\alpha$ expression shows some degree of correlation with the $\mathrm{IC}_{50}$ values obtained in our study (A549> A2780 > MCF7, M1, LoVo), suggesting a possible role for PKC $\alpha$ in the intrinsically resistant phenotype. Different PKC isoforms, and particularly $\mathrm{PKC} \alpha$, have been implicated in cell resistance to cytotoxic drugs and a number of putative substrates have been identified. One of the first to be examined was P-gp, and PKC $\alpha$ was proposed to enhance expression and/or function of this transporter in a number of cell lines $(26,27)$. However, our data seem to rule out P-gp as a relevant substrate, as DX accumulation was similar in all cell lines tested, in spite of different PKC $\alpha$ levels. Bcl-2 is another putative candidate, as phosphorylation by $\mathrm{PKC} \alpha$ has been reported to increase its anti-apoptotic effects (28). However, it is highly unlikely that Bcl-2 may be involved in this case, as A549 cells only express minimal amounts of this protein, whereas MCF7 cells exhibit high levels. In fact, A549 cells seem to rely on another Bcl-2 family member for protection against apoptosis, namely the $\mathrm{Bcl}-\mathrm{x}_{\mathrm{L}}$ protein (29). A recent report (30) indicates that $b c l$-x gene expression is modulated by $\mathrm{PKC} \alpha$; therefore the relationship between PKC $\alpha$ and $\mathrm{Bcl}-\mathrm{x}_{\mathrm{L}}$ in A549 cells may be worth investigating. Finally, there is some evidence that GST activity may also depend on PKCmediated phosphorylation (31).

In summary, in a small panel of human tumor cell lines we have examined a number of putative molecular determinants of drug response. Some of them, including classic multidrug transporters and apoptosis-related proteins, do not seem to affect drug response, or appear to be cell type-specific (LRP and GSH-related enzymes). In contrast, PKC $\alpha$ levels increase in parallel with drug resistance and may therefore play an as yet undefined role in determining drug response. Further investigations are under way to identify the relevant PKC substrates and possible strategies to inhibit their activation.

\section{References}

1. Larsen AK, Escargueil AE and Skladanowski A: Resistance mechanisms associated with altered intracellular distribution of anticancer agents. Pharmacol Ther 85: 217-229, 2000.

2. Ambudkar SV, Dey S, Hrycyna CA, Ramachandra M, Pastan I and Gottesman MM: Biochemical, cellular, and pharmacologica aspects of the multidrug transporter. Annu Rev Pharmacol Toxicol 39: 361-398, 1999.
3. Gottesman MM and Pastan I: Biochemistry of multidrug resistance mediated by the multidrug transporter. Annu Rev Biochem 62: 385-427, 1993.

4. Borst P, Evers R, Kool M and Wijnholds J: A family of drug transporters: the multidrug resistance-associated proteins. J Natl Cancer Inst 92: 1295-1302, 2000.

5. Laurencot CM, Scheffer GL, Scheper RJ and Shoemaker RH: Increased LRP mRNA expression is associated with the MDR phenotype in intrinsically resistant human cancer cell lines. Int J Cancer 72: 1021-1026, 1997

6. Berger W, Elbling L and Micksche M: Expression of major vault protein LRP in human non-small cell lung cancer cells: activation by short term exposure to antineoplastic drugs. Int $\mathbf{J}$ Cancer 88: 293-300, 2000.

7. Scheffer GL, Wijngaard PLJ, Flens MJ, Izquierdo MA, Slovack ML, Pinedo HM, Meijer CJLM, Clevers HC and Scheper RJ: The drug resistance-related protein LRP is the human major vault protein. Nat Med 1: 578-582, 1995.

8.Izquierdo MA, Scheffer GL, Flens MJ, Giaccone G, Broxterman HJ, Maijer CJ, van der Valk P and Scheper RJ: Broad distribution of the multidrug resistance-related vault lung resistance protein in normal human tissues and tumors. Am J Pathol 148: 877-887, 1996.

9.Tew KD: Glutathione-associated enzymes in anticancer drug resistance. Cancer Res 54: 4313-4320, 1994.

10. Kohn KW: Regulatory genes and drug sensitivity. J Natl Cancer Inst 88: 1255-1256, 1996.

11. Herr I and Debatin K-M: Cellular stress response and apoptosis in cancer therapy. Blood 98: 2603-2614, 2001.

12. Ruvolo PP, Deng X, Carr BK and May WS: A functional role for mitochondrial protein kinase $\mathrm{C} \alpha$ in $\mathrm{Bcl}-2$ phosphorylation and suppression of apoptosis. J Biol Chem 273: 25436-25442, 1998.

13. Porter AG: Protein translocation in apoptosis. Trends Cell Biol 9: 394-401, 1999.

14. Supino R: MTT assays. In: Methods in Molecular Biology. O'Hare SM and Atterwill CK (eds). Vol 43. Humana Press, Totowa, NJ, pp137-149, 1998.

15. Hissin PJ and Hilf R: A fluorimetric method for determination of oxidized and reduced glutathione in tissues. Anal Biochem 74: 214-226, 1976.

16. Habig WH, Pabst MJ and Jakoby WB: Glutathione S-transferase. J Biol Chem 249: 7130-7139, 1974.

17. Colman RF: Glutathione reductase (Yeast). Anal Biochem 14: 434-440, 1966.

18. Chou TC and Talalay P: Quantitative analysis of dose-effect relationships: the combined effects of multiple drugs or enzyme inhibitors. Adv Enzyme Regul 22: 27-55, 1984.

19. Meschini S, Marra M, Calcabrini A, Monti E, Gariboldi M, Dolfini E and Arancia G: Role of the lung resistance-related protein (LRP) in the drug sensitivity of cultured tumor cells. Toxicol In Vitro 16: 389-398, 2002.

20. Zaman GJ, Lankelma J, van Tellingen O, Beijnen J, Dekker H, Paulusma C, Oude Elferink RP, Baas F and Borst P: Role of glutathione in the export of compounds from cells by the multidrug-resistance-associated protein. Proc Natl Acad Sci USA 92: 7690-7694, 1995.

21. Rappa G, Lorico A, Flavell RA and Sartorelli AC: Evidence that the multidrug resistance protein (MRP) functions as a cotransporter of glutathione and natural product toxins. Cancer Res 57: 5232-5237, 1997.

22. Lai GM, Moscow JA, Alvarez MG, Fojo AT and Bates SE: Contribution of glutathione and glutathione-dependent enzymes in the reversal of adriamycin resistance in colon carcinoma cell lines. Int J Cancer 49: 688-695, 1991.

23. Zhang K, Yang EB, Wong KP and Mack P: GSH, GSH-related enzymes and GS-X pump in relation to sensitivity of human tumor cell lines to chlorambucil and adriamycin. Int J Oncol 14: 861-867, 1999.

24. Ban N, Takahashi Y, Takayama T, Kura T, Katahira T, Sakamaki S and Niitsu Y: Transfection of glutathione Stransferase (GST)-pi antisense complementary DNA increases the sensitivity of a colon cancer cell line to adriamycin, cisplatin, melphalan, and etoposide. Cancer Res 56: 35773582, 1996.

25. Beaumont PO, Moore MJ, Ahmad K, Payne MM, Lee C and Riddick DS: Role of glutathione S-transferases in the resistance of human colon cancer cell lines to doxorubicin. Cancer Res 58: 947-955, 1998. 
26. Gill PK, Gescher A and Gant TW: Regulation of MDR1 promoter activity in human breast carcinoma cells by protein kinase $C$ isozymes alpha and theta. Eur J Biochem 268: 41514157, 2001.

27. Germann UA, Chambers TC, Ambudkar SV, Pastan I and Gottesman MM: Effects of phosphorylation of P-glycoprotein on multidrug resistance. J Bioenerg Biomembr 27: 53-61, 1995.

28. Ito T, Deng X, Carr B and May WS: Bcl-2 phosphorylation required for anti-apoptotic function. J Biol Chem 272: 11671-11673, 1997.
29. Leech SH, Olie RA, Gautschi O, Simoes-Wust AP, Tschopp S, Haner R, Hall J, Stahel RA and Zangemeister-Wittke U: Induction of apoptosis in lung-cancer cells following bcl-xL anti-sense treatment. Int J Cancer 86: 570-576, 2000.

30. Leirdal $\mathrm{M}$ and Sioud M: Ribozyme inhibition of the protein kinase $\mathrm{C}$ alpha triggers apoptosis in glioma cells. Br J Cancer 80: 1558-1564, 1999.

31. Taniguchi $\mathrm{H}$ and Pyerin W: Glutathione-S-transferase is an in vitro substrate of $\mathrm{Ca}^{++}$-phospholipid-dependent protein kinase (protein kinase C). Biochem Biophys Res Commun 162: 903-907, 1989. 